\title{
CARPHOLOGY by Rajendra
}

Pract Neurol 2008; 8: 274

\section{Carotid bruits}

People with a carotid bruit are more likely to die from cardiovascular disease or have a heart attack than those without a bruit. This is the finding of a meta-analysis which included 17295 patients. The median sample size of the studies was 273 patients (range 38-4736) and the follow-up period was 4 years (range $2-7$ ). The rate of myocardial infarction in patients with carotid bruits was $3.69(95 \% \mathrm{Cl} 2.97$ to 5.40) per 100 patient-years compared with 1.86 (0.24 to 3.48) in those without bruits. Yearly rates of cardiovascular death were also higher in patients with bruits than in those without (2.85 (2.16 to 3.54) per 100 patient-years vs 1.11 (0.45 to 1.76)). Auscultation for carotid bruits in patients at risk for heart disease could help select those who might benefit the most from a strategy for modifying cardiovascular risk, say the authors.

Lancet 2008;371:1587-94.

\section{Brain haemorrhage}

Haemostatic therapy with activated factor VIla ( $\mathrm{rFVIIa)} \mathrm{reduced} \mathrm{the} \mathrm{growth} \mathrm{of} \mathrm{the}$ haematoma but did not improve survival or functional outcome after intracerebral hemorrhage. This is the finding of a trial which included 841 patients randomised to receive placebo, $20 \mu \mathrm{g}$ rFVlla per $\mathrm{kg}$ of body weight, or $80 \mu \mathrm{g}$ of rFVlla per $\mathrm{kg}$, within 4 hours after the onset of stroke. The growth in volume of intracerebral haemorrhage was reduced by $2.6 \mathrm{ml}(95 \% \mathrm{Cl}-0.3$ to 5.5 ; $\mathrm{p}=0.08$ ) in the group receiving $20 \mu \mathrm{g}$ of rFVlla per $\mathrm{kg}$ and by $3.8 \mathrm{ml}(95 \% \mathrm{Cl} 0.9$ to $6.7 ; p=0.009$ ) in the group receiving $80 \mu \mathrm{g}$, as compared with the placebo group. The overall frequency of thromboembolic serious adverse events was similar in the three groups though arterial events were more frequent in the group receiving $80 \mu \mathrm{g}$.

N Engl J Med 2008;358:2127-37.

\section{Stents or carotid endarterectomy?}

The three year results of the SAPPHIRE trial, in which patients with severe carotid artery stenosis and increased surgical risk underwent carotid artery stenting with an emboli-protection device or endarterectomy, show no significant differences between the two groups of patients. There were 15 strokes in each group, of which 11 in the stenting group and 9 in the endarterectomy group were ipsilateral. Data were available for 260 patients (78\%), including $86 \%$ of patients in the stenting group and $70 \%$ of those in the endarterectomy group. The short-term outcomes (30 days, 1 year) have been previously reported.

N Engl J Med 2008;358:1572-9.

\section{Steroids for bacterial meningitis in children}

Adjuvant corticosteroids significantly reduce mortality associated with bacterial meningitis in adults. Unfortunately they don't seem to have the same effect in children. This is the finding of a retrospective cohort study from the USA conducted over 6 years of 2780 children discharged with bacterial meningitis as their primary diagnosis. The median age of the patients was 9 months (interquartile range 0-6 years). Streptococcus pneumoniae was the most commonly identified cause of meningitis. Adjuvant corticosteroids were given to 248 children (8.9\%). The overall mortality was $4.2 \%(95 \% \mathrm{Cl} 3.5 \%$ to 5.0\%). Adjuvant corticosteroids did not reduce mortality nor did they reduce duration of stay in hospital.

JAMA 2008;299:2048-55.

\section{Carers of people with dementia}

Carers of people with dementia can feel lonely, and loneliness has been associated with increased mortality and physical and psychiatric morbidity. One would expect that providing a "befriender service" would improve the well being of a carer. Unfortunately, access to a befriender facilitator did not prove effective in improving carers' well-being or healthrelated quality of life. This is the conclusion of a single blind randomised controlled trial that included 236 family carers of people with primary progressive dementia. One reason for this could be that befriending schemes were not taken up by all carers.

BMJ doi:10.1136/bmj.39549.548831.AE

\section{Surgery for sciatica}

Early surgery achieved more rapid relief of sciatica than conservative care, but outcomes were similar by one year and these did not change during the second year. This is the finding of a randomised controlled trial to evaluate the effects of early lumbar disc surgery compared with prolonged conservative care. The trial included 238 patients with 6-12 weeks of sciatica who were followed up for two years. Improvement in leg pain was faster for patients randomised to early surgery but this short-term benefit was no longer significant by six months and continued to narrow between six months and 24 months. At two years 20\% of all patients reported an unsatisfactory outcome.

BMJ doi:10.1136/bmj.a143 